\title{
What Is the Ideal Blood Pressure Threshold for the Prevention of Atrial Fibrillation in Elderly General Population?
}

\author{
Yoon Jung Park ${ }^{1,+}$, Pil-Sung Yang ${ }^{2,+}{ }^{\oplus}$, Hee Tae Yu ${ }^{1}$, Tae-Hoon Kim ${ }^{1}$, Eunsun Jang ${ }^{1}{ }^{(}$, \\ Jae-Sun Uhm ${ }^{1} \mathbb{D}$, Hui-Nam Pak ${ }^{1}$, Moon-Hyoung Lee ${ }^{1}$, Gregory Y.H. Lip ${ }^{1,3, * \mathbb{D}}$ and \\ Boyoung Joung 1,*(D) \\ 1 Division of Cardiology, Department of Internal Medicine, Severance Cardiovascular Hospital, \\ Yonsei University College of Medicine, Seoul 03722, Korea; PYJ221@yuhs.ac (Y.J.P.); \\ HEETYU@yuhs.ac (H.T.Y.); THKIMCARDIO@yuhs.ac (T.-H.K.); SUNNY_JES@yuhs.ac (E.J.); \\ JASON@yuhs.ac (J.-S.U.); HNPAK@yuhs.ac (H.-N.P.); MHLEE@yuhs.ac (M.-H.L.) \\ 2 Department of Cardiology, CHA Bundang Medical Centre, CHA University, Seongnam 13496, Korea; \\ psyang01@cha.ac.kr \\ 3 Liverpool Centre for Cardiovascular Science, University of Liverpool and Liverpool Heart \& Chest Hospital, \\ Liverpool L14 3PE, UK \\ * Correspondence: Gregory.Lip@liverpool.ac.uk (G.Y.H.L.); cby6908@yuhs.ac (B.J.); Tel.: +82-2-2228-846 (B.J.) \\ + The first two authors contributed equally to this work.
}

Received: 25 August 2020; Accepted: 11 September 2020; Published: 16 September 2020

\begin{abstract}
Intensive blood pressure (BP) lowering in patients with hypertension at increased risk of cardiovascular disease has been associated with a lowered risk of incident atrial fibrillation (AF). It is uncertain whether maintaining the optimal BP levels can prevent AF in the general elderly population. We included 115,866 participants without AF in the Korea National Health Insurance Service-Senior ( $\geq 60$ years) cohort from 2002 to 2013. We compared the influence of BP on the occurrence of new-onset AF between octogenarians ( $\geq 80$ years) and non-octogenarians ( $<80$ years) subjects. With up to $6.7 \pm 1.7$ years of follow-up, 4393 incident AF cases occurred. After multivariable adjustment for potentially confounding clinical covariates, the risk of AF in non-octogenarians was significantly higher in subjects with BP levels of $<120 /<80$ and $\geq 140 / 90 \mathrm{~mm} \mathrm{Hg}$, with hazard ratios of 1.15 (95\% confidence interval (CI), 1.03-1.28; $p<0.001$ ) and 1.14 (95\% CI, 1.04-1.26; $p<0.001$ ), compared to the optimal BP levels $(120-129 /<80 \mathrm{~mm} \mathrm{Hg})$. In octogenarians, the optimal BP range was 130-139/80-89 $\mathrm{mm} \mathrm{Hg}$, higher than in non-octogenarians. A U-shaped relationship for the development of incident AF was evident in non-octogenarians, and BP levels of $120-129 /<80 \mathrm{~mm} \mathrm{Hg}$ were associated the lowest risk of incident AF. Compared to non-octogenarians, the lowest risk of AF was associated with higher BP levels of 130-139/80-89 mm Hg amongst octogenarians.
\end{abstract}

Keywords: atrial fibrillation; hypertension; elderly; prevention

\section{Introduction}

Hypertension is the most common comorbidity in patients with atrial fibrillation (AF) and is highly prevalent in patients with $\mathrm{AF}$, especially those aged over 60 years [1]. Elevated blood pressure (BP) is associated with a greater burden of AF [2] and every $20 \mathrm{~mm} \mathrm{Hg}$ increase in systolic blood pressure (SBP) has a $21 \%$ higher risk of AF [3]. The high incidence of hypertension with AF has prompted the argument that AF is another sign of hypertensive target organ damage [4-6].

Several epidemiological studies have shown that the levels of SBP 130-139 $\mathrm{mm} \mathrm{Hg}$ are also associated with increased risk of AF, compared to normal SBP $(<120 \mathrm{~mm} \mathrm{Hg})$ [7-10]. Several 
randomized controlled trials also showed the relationship between BP and risk of AF. In the Cardio-Sis trial (Controllo della Pressione Arteriosa Sistolica trial), the risk of new-onset AF was reduced in the tight control group (SBP $<130 \mathrm{~mm} \mathrm{Hg}$ ) compared to the usual control group (SBP < $140 \mathrm{~mm} \mathrm{Hg}$ ) in patients with hypertension without diabetes [11]. Other report have shown that the intensive therapy group (target SBP $<120 \mathrm{~mm} \mathrm{Hg}$ ) did not show statistical significance with respect to the incidence of $\mathrm{AF}$ in patients with hypertension and diabetes [12]. A recent study, using data from Systolic Blood Pressure Intervention Trial (SPRINT) found that intensive treatment with a target SBP of $<120 \mathrm{~mm}$ $\mathrm{Hg}$ in patients with hypertension at high risk of cardiovascular disease reduced the risk of AF [13]. However, the relationship of BP and incident AF has not been established in older subjects.

However, strict BP control can induce serious adverse events such as hypotension, syncope, electrolyte imbalance, and acute kidney injury [14]. The Elderly population is more likely have other risk factors and target organ damage that may be worsened by lowering BP than the younger population [15]. Exacerbation of postural hypotension could be associated with injurious falls, and a low BP targets could be related to an increased risk of reduced renal function amongst octogenarians (age $>80$ years) [16]. Of note, the 2018 European Society of Cardiology (ESC) and the European Society of Hypertension (ESH) guidelines recommend less strict BP control for the elderly (age $\geq 65$ years) and close monitoring of adverse effects. Additionally, a previous study suggests that intensive BP control had no more benefit than harm in patients with a 10 year-cardiovascular risk of $<18.2 \%$ [17].

It remains uncertain whether intensive BP lowering to a target SBP of $<120 \mathrm{~mm} \mathrm{Hg}$ results in further lowering of the risk of new-onset $\mathrm{AF}$ in octogenarians with hypertension. In this study using the nationwide population-based National Health Insurance Service (NHIS)-senior cohort (NHIS-Senior), we aimed to investigate the optimal $\mathrm{BP}$ levels for the prevention of incident $\mathrm{AF}$, defining the ideal $\mathrm{BP}$ threshold for the prevention of AF in the general elderly population. Second, we evaluated whether these associations were observed in different age groups and were influenced by strict BP control.

\section{Experimental Section}

Data were collected from the NHIS-Senior, which included about 558,147 individuals, accounting for approximately $10 \%$ of the total elderly population over 60 years old in South Korea (approximately 5.1 million) in 2002 [18]. The NHIS-Senior database included the following parameters: sociodemographic and socioeconomic information, insurance status, health checkup examinations, and records of patients' medical and dental history. These parameters have been stratified to cover 12 years (2002-2013) and anonymized in the cohort study to protect the privacy of individuals. This study was approved by the Institutional Review Board of Yonsei University Health System (4-2016-0179). Informed consent was waived. The NHIS-Senior database used in this study (NHIS-2016-2-171) was made by the NHIS of Korea. The authors declare no conflict of interest with the NHIS.

\subsection{BP Measurement}

BP measurements were obtained at local hospitals and clinics certified for medical health examination centers by the Korean National Health Insurance Corporation. After the patient rested for $5 \mathrm{~min}$ in the sitting position, brachial BP was measured by qualified medical personnel at each health examination center. A blood pressure (BP) measurement was repeated if the first measurement was $>120 / 80 \mathrm{~mm} \mathrm{Hg}$. Automatic oscillometric devices and mercury sphygmomanometers were used for BP measurements, with the choice of device being at the discretion of individual examination centers. The preferred recommendation stipulated the use of mercury sphygmomanometers until 2015, when the sale of mercury sphygmomanometers was banned. The average of the BP measured at the first and second medical examinations was used for analysis.

\subsection{Study Population}

From the Korean NHIS-Senior, a total of 312,736 patients who had a health checkup between 2005 and 2012 were enrolled, and follow-up data were reviewed until December 2013. The exclusion 
criteria were as follows: (i) patients who had AF before enrollment $(n=8873$ ); (ii) those who had heart failure (HF) before enrollment $(n=26,210)$; (iii) those who had ischemic stroke or transient ischemic attack before enrollment $(n=32,344)$; (iv) those who had myocardial infarction (MI) before enrollment ( $n=3944)$; (v) those who had hemorrhagic stroke before enrollment $(n=1149)$; (vi) those who had malignancy before enrollment $(n=25,436)$; (vii) those who had missing data $(n=120)$; and (viii) those who check BP once $(n=98,794)$. Finally, we included 115,866 patients with repeated BP measurement (Figure 1).

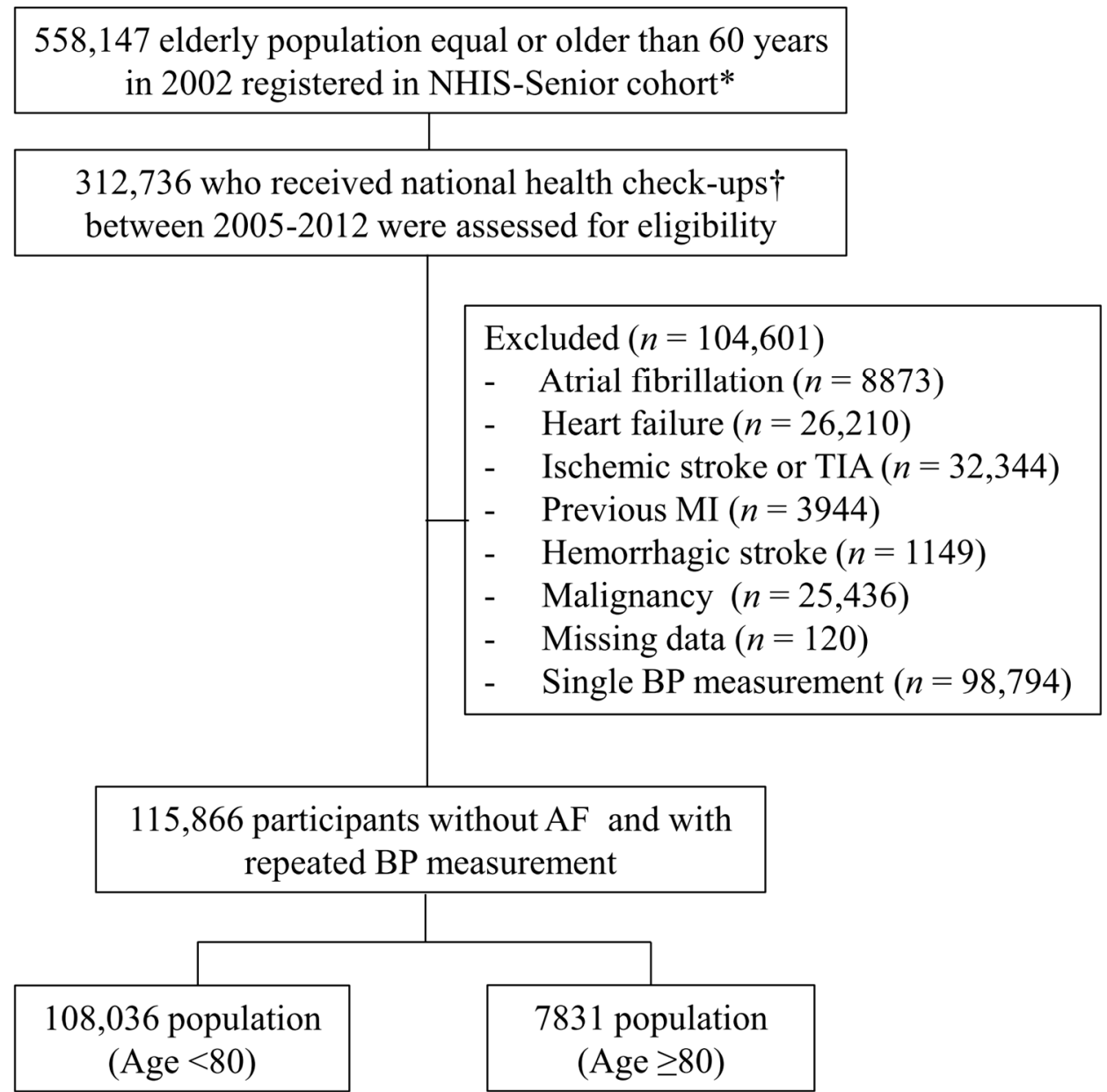

Figure 1. Flowchart of the study population enrollment and analyses. NHIS, National Health Insurance Service; TIA, transient ischemic attack; MI, myocardial infarction; BP, blood pressure; AF, atrial fibrillation. * Korean National Health Insurance Service (NHIS)-Senior cohort. + Complete checkup includes smoking, physical activity, alcohol, BMI, Total cholesterol, blood pressure, fasting glucose.

\subsection{Covariates}

We obtained information on selected comorbidities in inpatient and outpatient hospital diagnoses. Baseline comorbidities were defined using the medical claims and information about prescription medication prior to the index date. To ensure the accuracy of diagnosis, the patients were considered to have comorbid condition when the condition was a discharge diagnosis or confirmed at least twice in an outpatient setting according to previous studies using the NHIS (Supplementary Materials Table S1) $[19,20]$. For the status of standard income, the total amount of national health insurance premiums paid by the insured in the year was evaluated in proportion to personal income. 


\subsection{Hypertension and Atrial Fibrillation}

Hypertension was defined as the combination of previous hypertension diagnosis (International Classification of Disease-10th Revision (ICD-10) codes) and use of one or more antihypertensive drugs. The hypertension onset date for duration calculations was determined using information on the first date of hypertension diagnosis. The BP status was divided into four groups: (i) SBP of $<120 \mathrm{~mm} \mathrm{Hg}$ and diastolic blood pressure (DBP) of $<80 \mathrm{~mm} \mathrm{Hg}$; (ii) SBP of 120-129 $\mathrm{mm} \mathrm{Hg}$ and DBP of $<80 \mathrm{~mm} \mathrm{Hg}$; (iii) SBP of 130-139 mm Hg or DBP of 80-90 mm Hg; and (iv) SBP of $\geq 140 \mathrm{~mm} \mathrm{Hg}$ or DBP of $\geq 90 \mathrm{~mm}$ Hg. The study also compared the SBP status and incidence of AF. Furthermore, the relationship between the DBP status and incidence of AF was analyzed.

AF was diagnosed using the ICD-10, code I48. To ensure diagnostic accuracy, the patients were defined as having AF only when it was a discharge diagnosis or had been confirmed at least twice in the outpatient department. This AF diagnosis definition has been previously validated in the NHIS database with a positive predictive value of $94.1 \%[19,21]$.

\subsection{Statistical Analysis}

The baseline characteristics of participants with age over and under 80 years were compared using Student's $t$-test and Pearson's chi-square test. The incidence rates of events were calculated by dividing the number of events by person-times at risk, with the $95 \%$ confidence intervals (CI) estimated by exact Poisson distributions. Cox proportional hazards regressions were used to compare the incidence of AF with BP status. Two-sided $p$-values $<0.05$ were considered statistically significant. Statistical analyses were conducted using Statistical Package for Social Sciences (SPSS) version 23.0 (Chicago, IL, USA) and R version 3.3.2 (The R Foundation, www.R-project.org).

\section{Results}

\subsection{Baseline Characteristics}

Compared with non-octogenarians, the octogenarians were predominantly female and had more comorbidities, including hypertension, chronic kidney disease (CKD), anemia, chronic obstructive pulmonary disease, and osteoporosis (Table 1). The low rates of CKD and diabetes for ages in this study might be related with the rigid exclusion criteria of this study. The comparisons of baseline characteristics among patients with different BP levels in non-octogenarians and octogenarians are presented in Supplementary Materials Table S2.

Table 1. Comparison of clinical characteristics between the non-octogenarian and the octogenarian populations.

\begin{tabular}{ccccc}
\hline & All Population & \multicolumn{3}{c}{ Age } \\
\cline { 3 - 5 } & $(\mathbf{N}=\mathbf{1 1 5}, \mathbf{8 6 6})$ & $\begin{array}{c}\text { Age }<\mathbf{8 0} \\
(\boldsymbol{n = 1 0 8 , 0 3 5 )}\end{array}$ & $\begin{array}{c}\text { Age } \geq \mathbf{8 0} \\
(\boldsymbol{n}=\mathbf{7 8 3 1})\end{array}$ & $\boldsymbol{p}$-Value \\
\hline Age, years & $71.7(69.5-74.6)$ & $71.2(69.3-74.0)$ & $82.2(81.0-84.5)$ & $<0.001$ \\
Male & $53,609(46.3)$ & $50,391(46.6)$ & $3218(41.1)$ & $<0.001$ \\
Systolic BP & $130.5(122.5-140.0)$ & $130.0(122.5-140.0)$ & $132.5(124.5-142.5)$ & $<0.001$ \\
Diastolic BP * & $79.5(74.0-85.0)$ & $79.5(74.0-85.0)$ & $79.5(73.5-85.0)$ & 0.017 \\
Economic state * & $7.0(4.0-9.0)$ & $7.0(4.0-9.0)$ & $7.0(3.0-9.0)$ & 0.001 \\
\hline Alcohol & & & & $<0.001$ \\
No drinking & $64,681(80.9)$ & $60,075(80.6)$ & $4606(85.8)$ & \\
Moderate drinking & $5982(7.5)$ & $5673(7.6)$ & $309(5.8)$ & \\
Heavy drinking + & $9243(11.6)$ & $8789(11.8)$ & $454(8.5)$ & \\
\hline
\end{tabular}


Table 1. Cont.

\begin{tabular}{|c|c|c|c|c|}
\hline & \multirow{2}{*}{$\begin{array}{l}\text { All Population } \\
(N=115,866)\end{array}$} & \multicolumn{3}{|c|}{ Age } \\
\hline & & $\begin{array}{c}\text { Age }<80 \\
(n=108,035)\end{array}$ & $\begin{array}{l}\text { Age } \geq 80 \\
(n=7831)\end{array}$ & $p$-Value \\
\hline Smoking & & & & $<0.001$ \\
\hline $\begin{array}{l}\text { Non-smoker } \\
\text { or quit } \geq 12 \text { months }\end{array}$ & $63,389(79.3)$ & $58,993(79.1)$ & $4396(81.9)$ & \\
\hline Quit $<12$ months & $5732(7.2)$ & $5351(7.2)$ & $381(7.1)$ & \\
\hline Current smoker & $10,785(13.5)$ & $10,193(13.7)$ & $592(11.0)$ & \\
\hline \multicolumn{5}{|l|}{ Comorbidities } \\
\hline Hypertension & $46,519(40.1)$ & $42,883(39.7)$ & $3636(46.4)$ & $<0.001$ \\
\hline Diabetes & $14,767(12.7)$ & $13,881(12.8)$ & $886(11.3)$ & $<0.001$ \\
\hline Dyslipidemia & $34,200(29.5)$ & $32,369(30.0)$ & $1831(23.4)$ & $<0.001$ \\
\hline Chronic kidney disease & $997(0.9)$ & $913(0.8)$ & $84(1.1)$ & 0.041 \\
\hline Anemia & $17,715(15.3)$ & $15,715(14.6)$ & $2000(25.6)$ & $<0.001$ \\
\hline Hyperthyroidism & $2462(2.1)$ & $2341(2.2)$ & $121(1.5)$ & $<0.001$ \\
\hline Hypothyroidism & $2725(2.4)$ & $2583(2.4)$ & $142(1.8)$ & 0.001 \\
\hline COPD & $6960(6.0)$ & $6262(5.8)$ & $698(8.9)$ & $<0.001$ \\
\hline Liver disease & $23,559(20.3)$ & $22,335(20.7)$ & $1224(15.6)$ & $<0.001$ \\
\hline HCMP & $156(0.1)$ & $149(0.1)$ & $7(0.1)$ & 0.331 \\
\hline Osteoporosis & $33,139(28.6)$ & $30,569(28.3)$ & $2570(32.8)$ & $<0.001$ \\
\hline \multicolumn{5}{|l|}{ Medications } \\
\hline Aspirin & $19,315(16.7)$ & $17,957(16.6)$ & $1358(17.3)$ & 0.102 \\
\hline P2Y12 inhibitor & $872(0.8)$ & $816(0.8)$ & $56(0.7)$ & 0.742 \\
\hline ACE-inhibitor/ARB & $18,425(15.9)$ & $17,110(15.8)$ & $1315(16.8)$ & 0.027 \\
\hline Beta blocker & $18,115(15.6)$ & $16,815(15.6)$ & $1300(16.6)$ & 0.015 \\
\hline Calcium channel blocker & $30,370(26.2)$ & $27,945(25.9)$ & $2425(31.0)$ & $<0.001$ \\
\hline Statin & $11,918(10.3)$ & $11,333(10.5)$ & $585(7.5)$ & $<0.001$ \\
\hline Diuretics & $23,665(20.4)$ & $21,728(20.1)$ & $1937(24.7)$ & $<0.001$ \\
\hline MRA & $1677(1.4)$ & $1533(1.4)$ & $144(1.8)$ & 0.003 \\
\hline
\end{tabular}

BP, blood pressure; COPD, chronic obstructive pulmonary disease; HCMP, hypertrophic cardiomyopathy; ACE, angiotensin converting enzyme; $\mathrm{ARB}$, angiotensin II receptor blocker; MRA, mineralocorticoid receptor antagonist. * Several parameters including diastolic BP, economic state showed exactly the same median values. However, still significant (admittedly $p<0.05$ ) statistical differences because significantly different quartile values. $\dagger$ Male: $>112 \mathrm{~g} /$ week or $>42 \mathrm{~g} /$ day, Female: $>56 \mathrm{~g} /$ week or $>28 \mathrm{~g} /$ day. (14g per a glass). Values are presented as median (Q1-Q3 quartiles (25th and 75th percentiles)) or \%.

\subsection{BP and Incident AF in Different Age Groups}

During $6.4 \pm 2.1$ years of follow-up and a total of 768,314 person-years, 4393, 3946, and 447 incident $\mathrm{AF}$ cases occurred in the overall, non-octogenarian, and octogenarian populations, respectively. The spline curves of the SBP and DBP and risk of AF in different age groups are presented in Figure 2. A U-shaped relationship between SBP or DBP and risk of AF was evident; however, the U-shaped relationship for SBP was not observed in the octogenarian population. There is the larger uncertainty in the older group because it is numerically small. The optimal SBP level associated with the lowest the risks of AF was $120-129 \mathrm{~mm} \mathrm{Hg}$ in the overall population and the non-octogenarian population. The optimal DBP level with the lowest risk of AF was 70-79 $\mathrm{mm} \mathrm{Hg}$.

After multivariable adjustment for potentially confounding clinical covariates, in nonoctogenarians, the risk of AF was higher in patients with BP levels of $<120 /<80$ and $\geq 140 / 90 \mathrm{~mm} \mathrm{Hg}$ with adjusted hazard ratios (HR) of 1.15 (95\% CI, 1.03-1.28, $p<0.001$ ) and 1.14 (95\% CI, 1.04-1.26, $p<0.001$ ), respectively, compared to BP levels of $120-129 /<80 \mathrm{~mm} \mathrm{Hg}$. Amongst octogenarians, the risk of AF was significantly higher in patients with BP levels of $\geq 140 / 90 \mathrm{~mm} \mathrm{Hg}$ with an HR of 1.26 (95\% CI, 1.01-1.58, $p<0.001)$ compared with the optimal BP level (130-139/80-90 mm Hg; Table 2, Figure 3). 
(a)

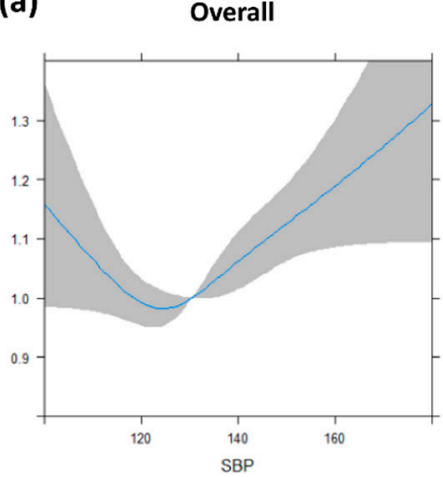

(b)

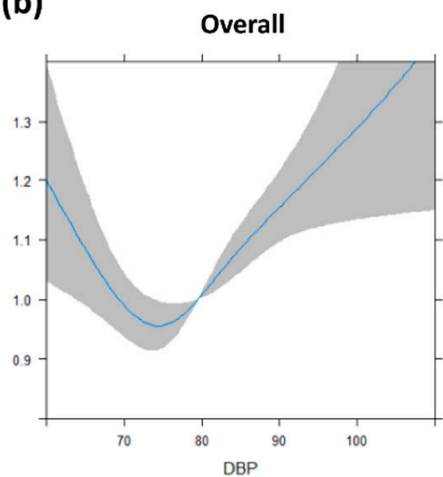

Age $<80$

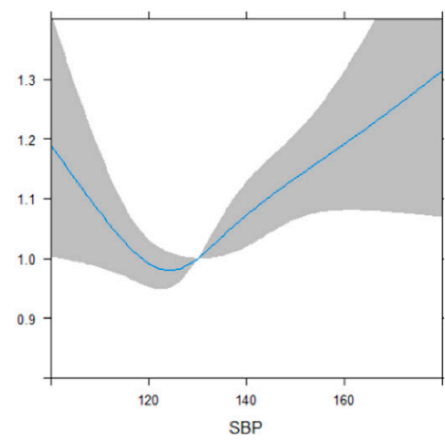

Age $<80$

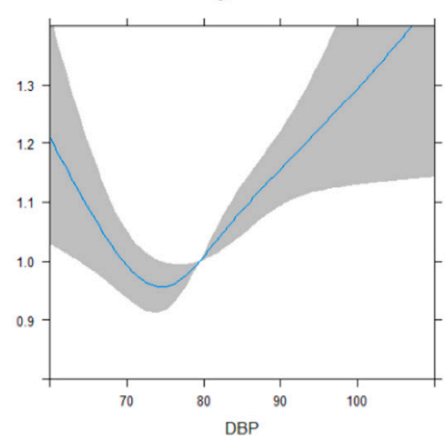

Age $\geq 80$

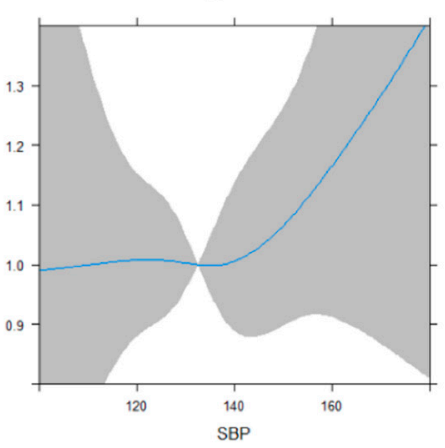

Age $\geq 80$

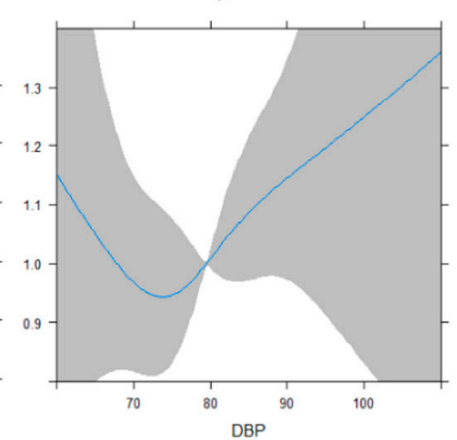

Figure 2. Systolic and diastolic blood pressure status of repeated measurement and risk of atrial fibrillation among elderly populations: (a) systolic blood pressure and (b) diastolic blood pressure. SBP, systolic blood pressure; DBP, diastolic blood pressure. The blue line shows relationship between hazard ratio of new-onset AF and blood pressure, and the gray area indicates the degree of confidence.

\section{(a)}

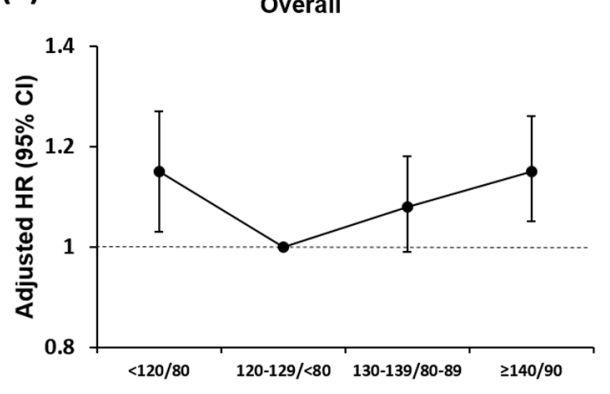

(b)

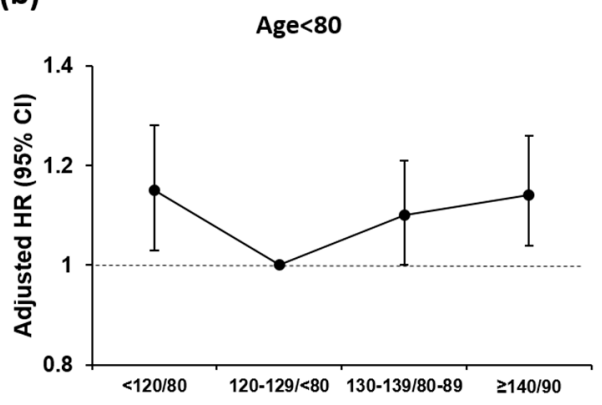

(c)

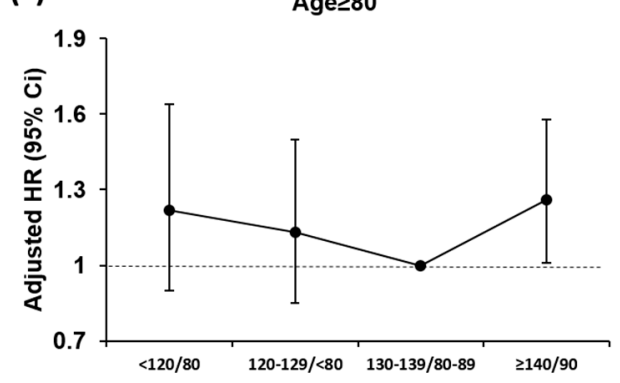

Figure 3. Blood pressure status of repeated measurement and risk of atrial fibrillation among elderly populations: (a) overall population, (b) age $<80$ years, and (c) age $\geq 80$ years. HR, hazard ratio. 
Table 2. Incidence Rate of Atrial Fibrillation According to Blood Pressure.

\begin{tabular}{|c|c|c|c|c|c|c|c|c|c|}
\hline \multirow[b]{2}{*}{ Group } & \multicolumn{3}{|c|}{ All Population } & \multicolumn{3}{|c|}{ Age $<80$ Years } & \multicolumn{3}{|c|}{ Age $\geq 80$ Years } \\
\hline & $\begin{array}{c}\text { No. } \\
\text { /Total No. } \\
4393 \\
/ 115,866\end{array}$ & $\begin{array}{c}\text { Incidence Rate } \\
\text { per } 1000 \\
\text { Person-Years } \\
(95 \mathrm{CI})\end{array}$ & $\begin{array}{l}\text { Hazard Ratio } \\
\quad(95 \mathrm{CI})\end{array}$ & $\begin{array}{c}\text { No. } \\
\text { /Total No. } \\
3946 \\
/ 108,035\end{array}$ & $\begin{array}{c}\text { Incidence Rate } \\
\text { Per } 1000 \\
\text { Person-Years } \\
\text { (95 CI) }\end{array}$ & $\begin{array}{l}\text { Hazard Ratio } \\
\text { (95 CI) }\end{array}$ & $\begin{array}{c}\text { No. } \\
\text { /Total No. } \\
447 \\
/ 7831\end{array}$ & $\begin{array}{c}\text { Incidence } \\
\text { Rate } \\
\text { Per } 1000 \\
\text { Person-Years } \\
\text { (95 CI) }\end{array}$ & $\begin{array}{l}\text { Hazard Ratio } \\
\text { (95 CI) }\end{array}$ \\
\hline $\begin{array}{l}<120 \\
\mid<80\end{array}$ & $\begin{array}{c}713 \\
/ 19,712\end{array}$ & $\begin{array}{c}5.47 \\
(5.07-5.88)\end{array}$ & $\begin{array}{c}1.15 \\
(1.03-1.27)\end{array}$ & $\begin{array}{c}649 \\
/ 18,556\end{array}$ & $\begin{array}{c}5.24 \\
(4.84-5.66)\end{array}$ & $\begin{array}{c}1.15 \\
(1.03-1.28)\end{array}$ & $\begin{array}{c}64 \\
/ 1156\end{array}$ & $\begin{array}{c}9.78 \\
(7.53-12.49)\end{array}$ & $\begin{array}{c}1.22 \\
(0.90-1.64)\end{array}$ \\
\hline $\begin{array}{c}120-129 \\
/<80\end{array}$ & $\begin{array}{c}721 \\
/ 21,463\end{array}$ & $\begin{array}{c}5.13 \\
(4.76-5.52)\end{array}$ & $\begin{array}{c}1 \\
\text { (reference) }\end{array}$ & $\begin{array}{c}647 \\
/ 20,104\end{array}$ & $\begin{array}{c}4.88 \\
(4.51-5.27)\end{array}$ & $\begin{array}{c}1 \\
\text { (reference) }\end{array}$ & $\begin{array}{c}74 \\
/ 1359\end{array}$ & $\begin{array}{c}9.37 \\
(7.35-11.76)\end{array}$ & $\begin{array}{c}1.13 \\
(0.85-1.50)\end{array}$ \\
\hline $\begin{array}{c}130-139 \\
/ 80-89\end{array}$ & $\begin{array}{c}1526 \\
/ 40,667\end{array}$ & $\begin{array}{c}5.67 \\
(5.39-5.96)\end{array}$ & $\begin{array}{c}1.08 \\
(0.99-1.18)\end{array}$ & $\begin{array}{c}1386 \\
/ 37,949\end{array}$ & $\begin{array}{c}5.47 \\
(5.19-5.77)\end{array}$ & $\begin{array}{c}1.10 \\
(1.00-1.21)\end{array}$ & $\begin{array}{c}140 \\
/ 2718\end{array}$ & $\begin{array}{c}8.65 \\
(7.28-10.21)\end{array}$ & $\begin{array}{c}1 \\
\text { (reference) }\end{array}$ \\
\hline $\begin{array}{l}\geq 140 \\
/ \geq 90\end{array}$ & $\begin{array}{c}1433 \\
/ 34,024\end{array}$ & $\begin{array}{c}6.28 \\
(5.96-6.62)\end{array}$ & $\begin{array}{c}1.15 \\
(1.05-1.26)\end{array}$ & $\begin{array}{c}1264 \\
/ 31,426\end{array}$ & $\begin{array}{c}5.95 \\
(5.63-6.29)\end{array}$ & $\begin{array}{c}1.14 \\
(1.04-1.26)\end{array}$ & $\begin{array}{c}169 \\
/ 2598\end{array}$ & $\begin{array}{c}10.76 \\
(9.20-12.52)\end{array}$ & $\begin{array}{c}1.26 \\
(1.01-1.58)\end{array}$ \\
\hline
\end{tabular}

CI, confidence interval. 


\subsection{BP and Incident AF in Patients with Treated Hypertension}

With a total of 298,087 person-years follow-up of patients with treated hypertension, there were 2069, 1846, and 3636 incident AF cases occurring in the overall, non-octogenarian, and octogenarian populations, respectively. The spline curves of the SBP and DBP and risk of AF in different age groups are presented in Figure 4 and had a similar pattern as the spline curve for the general populations (Figure 2).

(a)

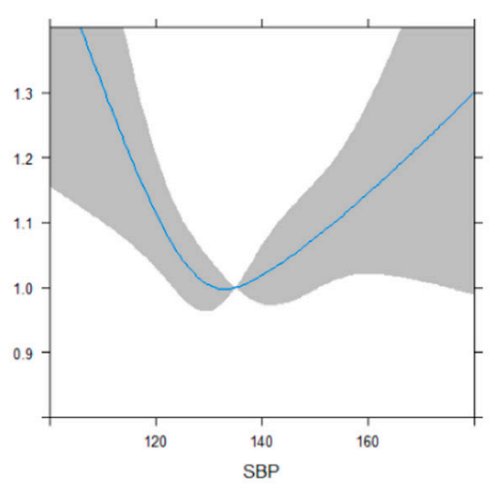

(b)

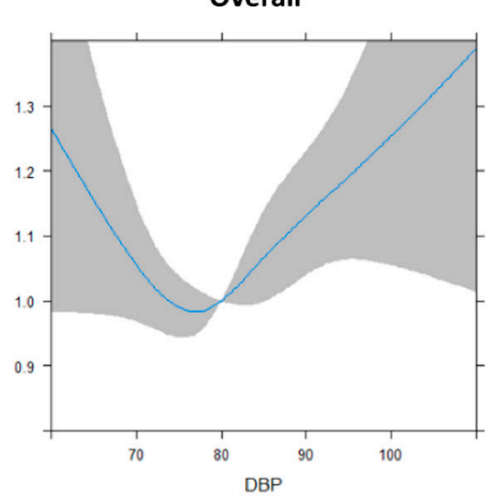

Age $<80$

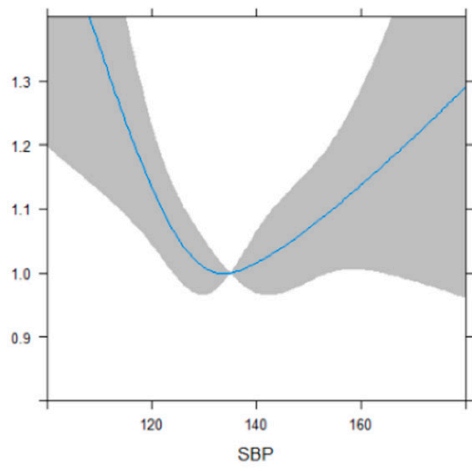

Age $<80$

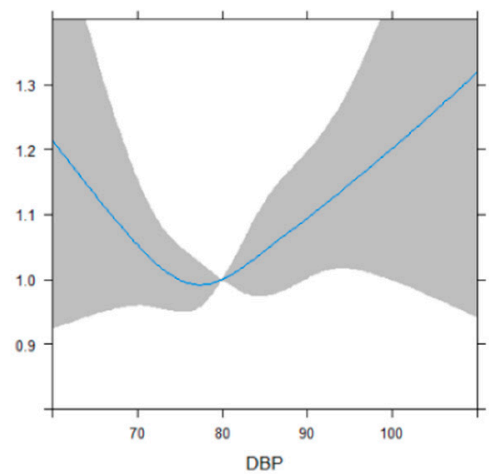

Age $\geq 80$

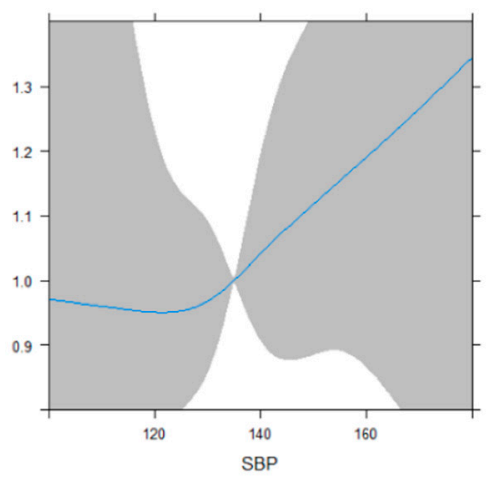

Age $\geq 80$

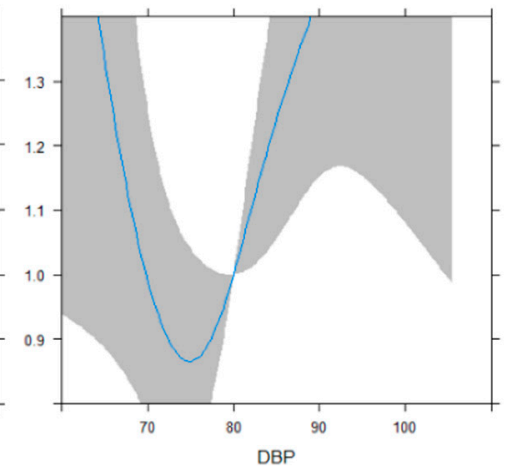

Figure 4. Systolic and diastolic blood pressure status of repeated measurement and risk of atrial fibrillation among elderly populations with antihypertensive medications: (a) systolic blood pressure and (b) diastolic blood pressure. SBP, systolic blood pressure; DBP, diastolic blood pressure. The blue line shows relationship between hazard ratio of new-onset AF and blood pressure, and the gray area indicates the degree of confidence.

After multivariable adjustment for potentially confounding clinical covariates in the non-octogenarian population, the risk of $\mathrm{AF}$ was higher in patients with intensive $\mathrm{BP}$ control $(<120 /<80 \mathrm{~mm} \mathrm{Hg})$ and poor $(\geq 140 / 90 \mathrm{~mm} \mathrm{Hg})$ BP control with adjusted HRs of $1.37(95 \% \mathrm{CI}$, $1.13-1.65, p<0.001)$ and $1.16(95 \% \mathrm{CI}, 1.0-1.33, p<0.001)$, respectively, compared to those with optimal $\mathrm{BP}$ control (120-129/<80 mm Hg). In octogenarians, the risk of AF was significantly higher in patients with a BP levels of $\geq 140 / 90 \mathrm{~mm} \mathrm{Hg}$ with an HR of 1.42 (95\% CI, 1.04-1.93, $p<0.001$ ) compared to those with optimal BP level (130-139/80-90 mm Hg; Table 3, Figure 5). 
Table 3. Incidence Rate of Atrial Fibrillation According to Blood Pressure in Patients with Treated Hypertension.

\begin{tabular}{|c|c|c|c|c|c|c|c|c|c|}
\hline & \multicolumn{3}{|c|}{ All Population } & \multicolumn{3}{|c|}{ Age $<80$ Years } & \multicolumn{3}{|c|}{ Age $\geq 80$ Years } \\
\hline Group & $\begin{array}{c}\text { No./ } \\
\text { Total No. } \\
2069 \\
/ 46,519\end{array}$ & $\begin{array}{c}\text { Incidence Rate } \\
\text { per } 1000 \\
\text { Person-Years } \\
\text { (95 CI) }\end{array}$ & $\begin{array}{l}\text { Hazard Ratio } \\
\text { (95 CI) }\end{array}$ & $\begin{array}{c}\text { No./ } \\
\text { Total No. } \\
1846 \\
/ 42,883\end{array}$ & $\begin{array}{c}\text { Incidence Rate } \\
\text { per } 1000 \\
\text { Person-Years } \\
(95 \mathrm{CI})\end{array}$ & $\begin{array}{l}\text { Hazard Ratio } \\
\text { (95 CI) }\end{array}$ & $\begin{array}{c}\text { No./ } \\
\text { Total No. } \\
223 \\
/ 3636\end{array}$ & $\begin{array}{c}\text { Incidence Rate } \\
\text { Per } 1000 \\
\text { Person-Years } \\
\text { (95 CI) }\end{array}$ & $\begin{array}{c}\text { Hazard Ratio } \\
\text { (95 CI) }\end{array}$ \\
\hline $\begin{array}{c}\text { Intensive } \\
\text { control } \\
(<120 / 80)\end{array}$ & $\begin{array}{c}210 \\
/ 4159\end{array}$ & $\begin{array}{c}8.22 \\
(7.14-9.41)\end{array}$ & $\begin{array}{c}1.34 \\
(1.13-1.61)\end{array}$ & $\begin{array}{c}189 \\
/ 3814\end{array}$ & $\begin{array}{c}7.96 \\
(6.87-9.18)\end{array}$ & $\begin{array}{c}1.37 \\
(1.13-1.65)\end{array}$ & $\begin{array}{c}21 \\
/ 345\end{array}$ & $\begin{array}{c}11.56 \\
(7.16-17.67)\end{array}$ & $\begin{array}{c}1.37 \\
(0.83-2.24)\end{array}$ \\
\hline $\begin{array}{c}\text { Optimal } \\
\text { control } \\
(120-129 \\
/<80)\end{array}$ & $\begin{array}{c}290 \\
/ 7466\end{array}$ & $\begin{array}{c}6.22 \\
(5.52-6.97)\end{array}$ & $\begin{array}{c}1 \\
\text { (reference) }\end{array}$ & $\begin{array}{c}257 \\
/ 6893\end{array}$ & $\begin{array}{c}5.91 \\
(5.21-6.68)\end{array}$ & $\begin{array}{c}1 \\
\text { (reference) }\end{array}$ & $\begin{array}{c}33 \\
/ 573\end{array}$ & $\begin{array}{c}10.36 \\
(7.13-14.55)\end{array}$ & $\begin{array}{c}1.17 \\
(0.77-1.79)\end{array}$ \\
\hline $\begin{array}{c}\text { Suboptimal } \\
\text { control } \\
(130-139 \\
/ 80-89)\end{array}$ & $\begin{array}{c}735 \\
/ 17,268\end{array}$ & $\begin{array}{c}6.66 \\
(6.19-7.16)\end{array}$ & $\begin{array}{c}1.09 \\
(0.95-1.25)\end{array}$ & $\begin{array}{c}667 \\
/ 15,934\end{array}$ & $\begin{array}{c}6.49 \\
(6.01-7.01)\end{array}$ & $\begin{array}{c}1.12 \\
(0.97-1.29)\end{array}$ & $\begin{array}{c}68 \\
/ 1334\end{array}$ & $\begin{array}{c}8.90 \\
(6.91-11.29)\end{array}$ & $\begin{array}{c}1 \\
\text { (reference) }\end{array}$ \\
\hline $\begin{array}{c}\text { Poor } \\
\text { control } \\
(\geq 140 / 90)\end{array}$ & $\begin{array}{c}834 \\
/ 17,626\end{array}$ & $\begin{array}{c}7.22 \\
(6.74-7.73)\end{array}$ & $\begin{array}{c}1.16 \\
(1.02-1.33)\end{array}$ & $\begin{array}{c}733 \\
/ 16,242\end{array}$ & $\begin{array}{c}6.83 \\
(6.34-7.34)\end{array}$ & $\begin{array}{c}1.16 \\
(1.00-1.33)\end{array}$ & $\begin{array}{c}101 \\
/ 1384\end{array}$ & $\begin{array}{c}12.46 \\
(10.15-15.14)\end{array}$ & $\begin{array}{c}1.42 \\
(1.04-1.93)\end{array}$ \\
\hline
\end{tabular}

CI, confidence interval. 


\section{(a)}

Overall

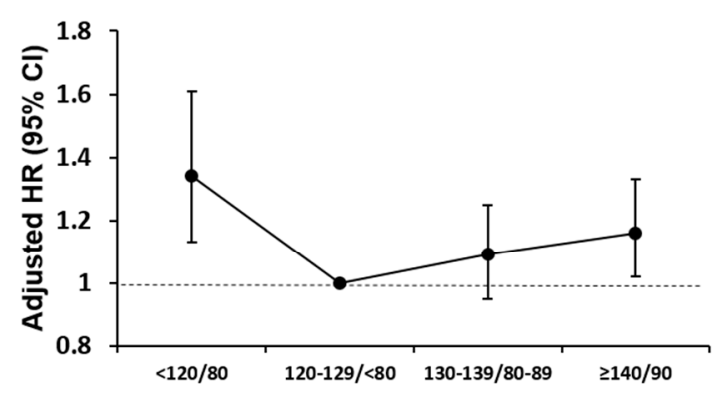

(b)

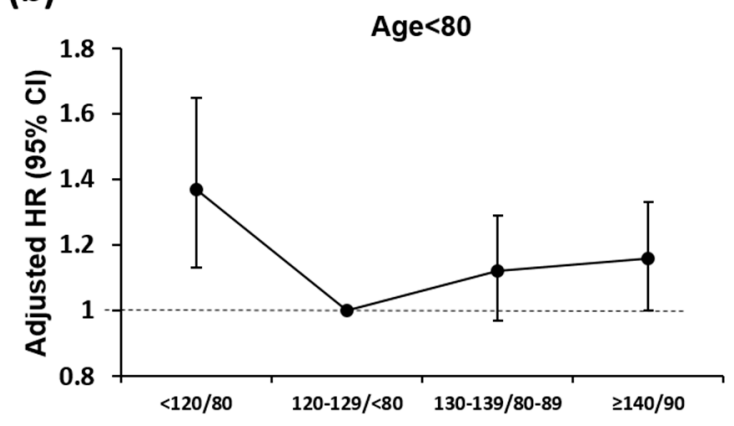

(c)

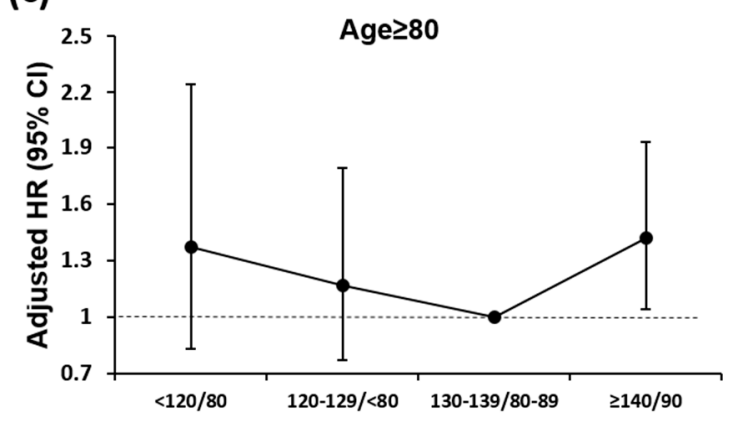

Figure 5. Blood pressure status of repeated measurement and risk of atrial fibrillation among elderly populations with antihypertensive medications: (a) overall population, (b) age $<80$ years, and (c) age $\geq 80$ years.

\subsection{Serious Adverse Events according to BP Status in Different Age Groups}

The incidence rate and HR of serious adverse events according to BP status in patients with hypertension treatment are presented in Table 4. In octogenarians, patients with intensive BP control $(<120 /<80 \mathrm{~mm} \mathrm{Hg})$ showed more hypotension requiring hospitalization than those with a BP levels of 130-139/80-90 $\mathrm{mm} \mathrm{Hg}$ with an adjusted HR of 2.06 (95\% CI, 1.12-3.81, $p<0.001$ ). The composite adverse events (including hypotension requiring hospitalization, syncope, bradycardia, electrolyte abnormality, injurious falls, and acute kidney injury) were numerically more frequent but non-statistically significant in patients with intensive and optimal BP control compared to those with a BP level of 130-139/80-89 mm Hg. 
Table 4. Incidence Rate and Hazard Ratio of Serious Adverse Events According to Blood Pressure Status in Patients with Hypertension Treatment.

\begin{tabular}{|c|c|c|c|c|c|}
\hline & & \multicolumn{4}{|c|}{ Blood Pressure Status } \\
\hline & & $<120 / 80 \mathrm{mmHg}$ & $120-129 /<80 \mathrm{mmHg}$ & $130-139 / 80-89 \mathrm{mmHg}$ & $\geq 140 / 90 \mathrm{mmHg}$ \\
\hline Overall & No. of total & 4159 & 7466 & 17,268 & 17,626 \\
\hline Composite event & $\begin{array}{l}\text { No. of events, } \\
\text { HR (95 CI) }\end{array}$ & $\begin{array}{c}341 \\
1.08(0.94-1.23)\end{array}$ & $\begin{array}{c}580 \\
1 \text { (reference) }\end{array}$ & $\begin{array}{c}1269 \\
0.95(0.86-1.04)\end{array}$ & $\begin{array}{c}1437 \\
1.01(0.92-1.11)\end{array}$ \\
\hline $\begin{array}{l}\text { Hypotension requiring } \\
\text { hospitalization }\end{array}$ & $\begin{array}{l}\text { No. of events, } \\
\text { HR (95 CI) }\end{array}$ & $\begin{array}{c}95 \\
1.15(0.89-1.48)\end{array}$ & $\begin{array}{c}151, \\
1 \text { (reference) }\end{array}$ & $\begin{array}{c}348 \\
0.99(0.82-1.20)\end{array}$ & $\begin{array}{c}366, \\
0.98(0.81-1.19)\end{array}$ \\
\hline Syncope & $\begin{array}{l}\text { No. of events } \\
\text { HR }(95 \mathrm{CI})\end{array}$ & $\begin{array}{c}33 \\
1.08(0.70-1.67)\end{array}$ & $\begin{array}{c}55, \\
1 \text { (reference) }\end{array}$ & $\begin{array}{c}155 \\
1.23(0.90-1.67)\end{array}$ & $\begin{array}{c}157 \\
1.18(0.86-1.60)\end{array}$ \\
\hline Bradycardia & $\begin{array}{l}\text { No. of events } \\
\text { HR }(95 \mathrm{CI})\end{array}$ & $\begin{array}{c}22 \\
0.99(0.59-1.67)\end{array}$ & $\begin{array}{c}41 \\
1 \text { (reference) }\end{array}$ & $\begin{array}{c}96 \\
1.01(0.70-1.45)\end{array}$ & $\begin{array}{c}97 \\
0.96(0.67-1.39)\end{array}$ \\
\hline Electrolyte abnormality & $\begin{array}{l}\text { No. of events } \\
\text { HR }(95 \mathrm{CI})\end{array}$ & $\begin{array}{c}142 \\
0.99(0.80-1.21)\end{array}$ & $\begin{array}{c}263 \\
1 \text { (reference) }\end{array}$ & $\begin{array}{c}523 \\
0.86(0.75-1.00)\end{array}$ & $\begin{array}{c}646 \\
1.01(0.88-1.17)\end{array}$ \\
\hline Injurious falls & $\begin{array}{l}\text { No. of events } \\
\text { HR (95 CI) }\end{array}$ & $\begin{array}{c}11 \\
1.11(0.52-2.37)\end{array}$ & $\begin{array}{c}17 \\
1 \text { (reference) }\end{array}$ & $\begin{array}{c}46 \\
1.18(0.67-2.06)\end{array}$ & $\begin{array}{c}41 \\
0.97(0.55-1.71)\end{array}$ \\
\hline Acute kidney injury & $\begin{array}{l}\text { No. of events } \\
\text { HR (95 CI) }\end{array}$ & $\begin{array}{c}106 \\
1.18(0.93-1.50)\end{array}$ & $\begin{array}{c}172 \\
1 \text { (reference) }\end{array}$ & $\begin{array}{c}364 \\
0.90(0.75-1.08)\end{array}$ & $\begin{array}{c}433 \\
0.96(0.80-1.14)\end{array}$ \\
\hline Age $<80$ Years & No. of total & 3814 & 6893 & 15,934 & 16,242 \\
\hline Composite event & $\begin{array}{l}\text { No. of events } \\
\text { HR (95 CI) }\end{array}$ & $\begin{array}{c}298 \\
1.08(0.94-1.25)\end{array}$ & $\begin{array}{c}506 \\
1 \text { (reference) }\end{array}$ & $\begin{array}{c}1124 \\
0.96(0.87-1.07)\end{array}$ & $\begin{array}{c}1279 \\
1.03(0.93-1.15)\end{array}$ \\
\hline $\begin{array}{l}\text { Hypotension requiring } \\
\text { hospitalization }\end{array}$ & $\begin{array}{l}\text { No. of events } \\
\text { HR (95 CI) }\end{array}$ & $\begin{array}{c}80 \\
1.13(0.85-1.49)\end{array}$ & $\begin{array}{c}129 \\
1 \text { (reference) }\end{array}$ & $\begin{array}{c}313 \\
1.05(0.85-1.28)\end{array}$ & $\begin{array}{c}327 \\
1.03(0.84-1.27)\end{array}$ \\
\hline Syncope & $\begin{array}{l}\text { No. of events } \\
\text { HR (95 CI) }\end{array}$ & $\begin{array}{c}31 \\
1.28(0.81-2.02)\end{array}$ & $\begin{array}{c}44 \\
1 \text { (reference) }\end{array}$ & $\begin{array}{c}141 \\
1.39(0.99-1.96)\end{array}$ & $\begin{array}{c}143 \\
1.33(0.95-1.87)\end{array}$ \\
\hline Bradycardia & $\begin{array}{l}\text { No. of events } \\
\text { HR (95 CI) }\end{array}$ & $\begin{array}{c}18 \\
0.88(0.50-1.55)\end{array}$ & $\begin{array}{c}38 \\
1 \text { (reference) }\end{array}$ & $\begin{array}{c}91 \\
1.03(0.71-1.51)\end{array}$ & $\begin{array}{c}87 \\
\text { 0.9. }(0.63-1.36) \\
\end{array}$ \\
\hline Electrolyte abnormality & $\begin{array}{c}\text { No. of events } \\
\text { HR (95 CI) }\end{array}$ & $\begin{array}{c}122 \\
0.98(0.79-1.22)\end{array}$ & $\begin{array}{c}228 \\
1 \text { (reference) }\end{array}$ & $\begin{array}{c}453 \\
0.87(0.74-1.02)\end{array}$ & $\begin{array}{c}573 \\
1.04(0.8-1.22)\end{array}$ \\
\hline
\end{tabular}


Table 4. Cont.

\begin{tabular}{|c|c|c|c|c|c|}
\hline & & \multicolumn{4}{|c|}{ Blood Pressure Status } \\
\hline & & $<120 / 80 \mathrm{mmHg}$ & $120-129 /<80 \mathrm{mmHg}$ & $130-139 / 80-89 \mathrm{mmHg}$ & $\geq 140 / 90 \mathrm{mmHg}$ \\
\hline \multirow{2}{*}{ Injurious falls } & No. of events & 10 & 17 & 40 & 38 \\
\hline & HR (95 CI) & $1.02(0.45-2.24)$ & 1 (reference) & $1.04(0.59-1.84)$ & $0.91(0.51-1.61)$ \\
\hline \multirow{2}{*}{ Acute kidney injury } & No. of events & 96 & 148 & 317 & 378 \\
\hline & HR (95 CI) & $1.25(0.96-1.61)$ & 1 (reference) & $0.91(0.75-1.11)$ & $0.98(0.81-1.18)$ \\
\hline Age $\geq 80$ Years & No of total & 345 & 573 & 1334 & 1384 \\
\hline \multirow{2}{*}{ Composite event } & No. of events & 43 & 74 & 145 & 158 \\
\hline & HR (95 CI) & $1.32(0.94-1.86)$ & $1.25(0.94-1.66)$ & 1 (reference) & $1.03(0.82-1.29)$ \\
\hline \multirow{2}{*}{$\begin{array}{l}\text { Hypotension requiring } \\
\text { hospitalization }\end{array}$} & No. of events & 15 & 22 & 35 & 39 \\
\hline & HR (95 CI) & $2.06(1.12-3.81)$ & $1.64(0.96-2.81)$ & 1 (reference) & $1.08(0.68-1.72)$ \\
\hline \multirow{2}{*}{ Syncope } & No. of events & 2 & 11 & 14 & 14 \\
\hline & HR (95 CI) & $0.57(0.13-2.51)$ & $1.69(0.74-3.85)$ & 1 (reference) & $1.69(0.74-3.85)$ \\
\hline \multirow{2}{*}{ Bradycardia } & No. of events & 4 & 3 & 5 & 10 \\
\hline & HR (95 CI) & $3.73(0.97-14.32)$ & $0.98(0.19-5.14)$ & 1 (reference) & $2.02(0.68-6.01)$ \\
\hline \multirow{2}{*}{ Electrolyte abnormality } & No. of events & 20 & 35 & 70 & 73 \\
\hline & HR (95 CI) & $1.21(0.73-1.99)$ & $1.18(0.78-1.79)$ & 1 (reference) & $0.98(0.70-1.36)$ \\
\hline \multirow{2}{*}{ Injurious falls } & No. of events & 1 & 0 & 6 & 3 \\
\hline & HR (95 CI) & $0.75(0.09-5.90)$ & 0 & 1 (reference) & $0.44(0.11-1.72)$ \\
\hline \multirow{2}{*}{ Acute kidney Injury } & No. of events & 10 & 24 & 47 & 55 \\
\hline & HR (95 CI) & $1.06(0.53-2.11)$ & $1.27(0.77-2.11)$ & 1 (reference) & $1.02(0.69-1.52)$ \\
\hline
\end{tabular}

CI, confidence interval. 


\section{Discussion}

In this large nationwide study on the impact of hypertension on incident AF, our principal findings are that BP levels of $120-129 /<80 \mathrm{~mm} \mathrm{Hg}$ were associated with a lower risk of incident AF in non-octogenarians. Amongst octogenarians, an average $10 \mathrm{~mm}$ Hg higher BP level of 130-139/80-89 mm $\mathrm{Hg}$ was more optimal to prevent AF. Second, octogenarians with intensive BP control $(<120 /<80 \mathrm{~mm}$ $\mathrm{Hg}$ ) showed more hypotension requiring hospitalization compared with BP level of 130-139/80-90 mm $\mathrm{Hg}$. Hence, a less strict BP level may be better to prevent AF and adverse effects amongst octogenarians.

The relationship between high $\mathrm{BP}$ and high incidence of $\mathrm{AF}$ supports the importance of $\mathrm{BP}$ control to prevent $\mathrm{AF}$ in patients with hypertension. Hypertension is the most common and important modifiable AF risk factor $[2,3,22,23]$. In a SPRINT sub-analysis, intensive BP control targeting $\mathrm{SBP}<120 \mathrm{~mm} \mathrm{Hg}$ was related with a lower risk of new-onset AF [13]. In contrast, the present study found a U-shaped relationship was observed between $\mathrm{BP}$ and incident $\mathrm{AF}$ in both non-octogenarians and octogenarians. This U-shaped relationship could be related to several unique aspects of our study cohort.

The present study recruited participants aged over 60 years, with the median age of our population being 71.7 years, much older than previous studies [11-13], and $6.8 \%$ of overall population were individuals of age over 80 years. The elderly population had more comorbidities, and these factors may influence the U-shaped relationship. Since the incidence of AF increases with age, we evaluated the relationship between the optimal BP and AF risk for BP management in older individuals. When comparing the relationship between AF and DBP, a U-shaped pattern was also observed in the overall population and patients with hypertension.

\subsection{Optimal BP Levels and Incident AF in Octogenarians}

Amongst octogenarians, an average $10 \mathrm{~mm} \mathrm{Hg}$ higher BP level of 130-139/80-89 mm Hg was more optimal to prevent AF; this compares to non-octogenarians where the optimal BP level was $120-129 /<80 \mathrm{~mm} \mathrm{Hg}$. However, the management of hypertension in octogenarians offers more challenges than in non-octogenarians. Elderly patients (age $>80$ years) have more comorbidities and higher risks other organ damage than patients aged under 80 years. In old patients, physicians should consider the risks and benefits when controlling BP due to aggravation of postural hypotension and reduction of renal function [16]. Also, intensive BP control has been related to increased serious adverse events such as hypotension, syncope, electrolyte abnormalities, and acute kidney injury [14]. Our results show an increased risk of hypotension requiring hospitalization in the intensive BP control group (BP < 120/80 mm Hg) compared with BPs 130-139/80-89 mm Hg. Even though other adverse events did not show significant differences, the composite outcome of adverse events showed a trend towards an increased risk in patients with intensive control (BP $<120 / 80 \mathrm{~mm} \mathrm{Hg}$ ) and optimal BP control (120-129/<80 mm Hg) compared to patients with BP levels of 130-139/80-89 mm Hg.

\subsection{Limitations}

The study has several limitations. First, in such studies using administrative databases, coding inaccuracies can lead to errors. Hence, we applied the definition that we had already validated in previous studies to minimize the problem [21,24-28]. Second, since the health examination of individuals was conducted in different hospitals and clinics, a uniformity of BP measurement could not be achieved. Third, the arbitrary cut-offs across continuous distributions (e.g., age, BP) were used to compare groups using simple binary statistical tests in this study. While the simplification can illustrate possible trends, it does automatically lead to a loss of detail in data analysis. Fourth, those who survive into their ninth decade are already a positively selected group and presumably with useful healthy characteristics. Fifth, since data about the types of AF and differential diagnosis between AF and atrial flutter (AFL) were not available, we could not investigate about the difference of optimal BP level according to the types of AF or AFL. Finally, hypertension and AF are associated with renal dysfunction. In our study, CKD was defined using the medical record with ICD-10 codes. There was 
no data on proteinuria. The lack of data on proteinuria, which is one of the criteria for CKD, may lead to low accuracy in defining CKD. This is one of the limitations of the study. Despite these limitations, the study is the first assessment to investigate the association between BP levels and incidental AF in a nationwide elderly population.

\section{Conclusions}

A U-shaped relationship for the development of incident AF was evident in non-octogenarians, and BP levels of 120-130/<80 mm Hg were associated the lowest risk of incident AF. Compared to non-octogenarians, the lowest risk of AF was associated with higher BP levels of 130-139/80-89 mm Hg amongst octogenarians.

Supplementary Materials: The following are available online at http://www.mdpi.com/2077-0383/9/9/2988/s1; Supplementary Table S1: Definitions and International Classification of Disease-10th Revision (ICD-10) codes used for defining the comorbidities and clinical outcomes; Supplementary Table S2: Comparison of baseline characteristics in patients with different blood pressure levels in overall population.

Author Contributions: Conceptualization, B.J. and G.Y.H.L.; methodology, P.-S.Y.; software, Y.J.P.; validation, E.J., H.T.Y., and T.-H.K.; formal analysis, J.-S.U.; investigation, Y.J.P.; resources, P.-S.Y.; data curation, H.-N.P.; writing - original draft preparation, Y.J.P.; writing—review and editing, G.Y.H.L.; visualization, M.-H.L.; supervision, M.-H.L.; project administration, B.J.; funding acquisition, B.J. All authors have read and agreed to the published version of the manuscript.

Funding: This research was funded by a research grant from the Korean Healthcare Technology R\&D project funded by the Ministry of Health and Welfare (HI15C1200, HC19C0130) and a CMB-Yuhan research grant of Yonsei University College of Medicine (6-2019-0124).

Acknowledgments: The National Health Information Database was provided by the National Health Insurance Service of Korea. We thank the National Health Insurance Service for its cooperation.

Conflicts of Interest: G.Y.H.L.: Consultant for Bayer/Janssen, BMS/Pfizer, Biotronik, Medtronic, Boehringer Ingelheim, Microlife, and Daiichi-Sankyo. Speaker for Bayer, BMS/Pfizer, Medtronic, Boehringer Ingelheim, Microlife, Roche, and Daiichi-Sankyo. No fees were received personally. B.J.: Speaker for Bayer, BMS/Pfizer, Medtronic, and Daiichi-Sankyo and research funds from Medtronic and Abbott. No fees were directly received personally. None of the other authors have anything to disclose.

\section{References}

1. Kearney, P.M.; Whelton, M.; Reynolds, K.; Muntner, P.; Whelton, P.K.; He, J. Global burden of hypertension: Analysis of worldwide data. Lancet 2005, 365, 217-223. [CrossRef]

2. Huxley, R.R.; Lopez, F.L.; Folsom, A.R.; Agarwal, S.K.; Loehr, L.R.; Soliman, E.Z.; Maclehose, R.; Konety, S.; Alonso, A. Absolute and attributable risks of atrial fibrillation in relation to optimal and borderline risk factors: The Atherosclerosis Risk in Communities (ARIC) study. Circulation 2011, 123, 1501-1508. [CrossRef] [PubMed]

3. Emdin, C.A.; Anderson, S.G.; Salimi-Khorshidi, G.; Woodward, M.; MacMahon, S.; Dwyer, T.; Rahimi, K. Usual blood pressure, atrial fibrillation and vascular risk: Evidence from 4.3 million adults. Int. J. Epidemiol. 2017, 46, 162-172. [CrossRef]

4. Dzeshka, M.S.; Shantsila, A.; Shantsila, E.; Lip, G.Y.H. Atrial Fibrillation and Hypertension. Hypertension 2017, 70, 854-861. [CrossRef] [PubMed]

5. Gumprecht, J.; Domek, M.; Lip, G.Y.H.; Shantsila, A. Invited review: Hypertension and atrial fibrillation: Epidemiology, pathophysiology, and implications for management. J. Hum. Hypertens. 2019, 33, 824-836. [CrossRef] [PubMed]

6. Lip, G.Y.H.; Coca, A.; Kahan, T.; Boriani, G.; Manolis, A.S.; Olsen, M.H.; Oto, A.; Potpara, T.S.; Steffel, J.; Marin, F; et al. Hypertension and cardiac arrhythmias: A consensus document from the European Heart Rhythm Association (EHRA) and ESC Council on Hypertension, endorsed by the Heart Rhythm Society (HRS), Asia-Pacific Heart Rhythm Society (APHRS) and Sociedad Latinoamericana de Estimulacion Cardiaca y Electrofisiologia (SOLEACE). Europace 2017, 19, 891-911. [CrossRef] [PubMed]

7. O'Neal, W.T.; Soliman, E.Z.; Qureshi, W.; Alonso, A.; Heckbert, S.R.; Herrington, D. Sustained pre-hypertensive blood pressure and incident atrial fibrillation: The Multi-Ethnic Study of Atherosclerosis. J. Am. Soc Hypertens. 2015, 9, 191-196. [CrossRef] 
8. Conen, D.; Tedrow, U.B.; Koplan, B.A.; Glynn, R.J.; Buring, J.E.; Albert, C.M. Influence of systolic and diastolic blood pressure on the risk of incident atrial fibrillation in women. Circulation 2009, 119, $2146-2152$. [CrossRef]

9. Grundvold, I.; Skretteberg, P.T.; Liestol, K.; Erikssen, G.; Kjeldsen, S.E.; Arnesen, H.; Erikssen, J.; Bodegard, J. Upper normal blood pressures predict incident atrial fibrillation in healthy middle-aged men: A 35-year follow-up study. Hypertension 2012, 59, 198-204. [CrossRef]

10. Thomas, M.C.; Dublin, S.; Kaplan, R.C.; Glazer, N.L.; Lumley, T.; Longstreth, W.T., Jr.; Smith, N.L.; Psaty, B.M.; Siscovick, D.S.; Heckbert, S.R. Blood pressure control and risk of incident atrial fibrillation. Am. J. Hypertens. 2008, 21, 1111-1116. [CrossRef]

11. Verdecchia, P.; Staessen, J.A.; Angeli, F.; de Simone, G.; Achilli, A.; Ganau, A.; Mureddu, G.; Pede, S.; Maggioni, A.P.; Lucci, D.; et al. Usual versus tight control of systolic blood pressure in non-diabetic patients with hypertension (Cardio-Sis): An open-label randomised trial. Lancet 2009, 374, 525-533. [CrossRef]

12. Chen, L.Y.; Bigger, J.T.; Hickey, K.T.; Chen, H.; Lopez-Jimenez, C.; Banerji, M.A.; Evans, G.; Fleg, J.L.; Papademetriou, V.; Thomas, A.; et al. Effect of Intensive Blood Pressure Lowering on Incident Atrial Fibrillation and P-Wave Indices in the ACCORD Blood Pressure Trial. Am. J. Hypertens. 2016, 29, 1276-1282. [CrossRef] [PubMed]

13. Soliman, E.Z.; Rahman, A.F.; Zhang, Z.M.; Rodriguez, C.J.; Chang, T.I.; Bates, J.T.; Ghazi, L.; Blackshear, J.L.; Chonchol, M.; Fine, L.J.; et al. Effect of Intensive Blood Pressure Lowering on the Risk of Atrial Fibrillation. Hypertension 2020, 75, 1491-1496. [CrossRef] [PubMed]

14. Group, S.R.; Wright, J.T., Jr.; Williamson, J.D.; Whelton, P.K.; Snyder, J.K.; Sink, K.M.; Rocco, M.V.; Reboussin, D.M.; Rahman, M.; Oparil, S.; et al. A Randomized Trial of Intensive versus Standard Blood-Pressure Control. N. Engl. J. Med. 2015, 373, 2103-2116. [CrossRef]

15. Williams, B.; Mancia, G.; Spiering, W.; Agabiti Rosei, E.; Azizi, M.; Burnier, M.; Clement, D.L.; Coca, A.; de Simone, G.; Dominiczak, A.; et al. 2018 ESC/ESH Guidelines for the management of arterial hypertension. Eur. Heart J. 2018, 39, 3021-3104. [CrossRef]

16. Kjeldsen, S.E.; Stenehjem, A.; Os, I.; Van de Borne, P.; Burnier, M.; Narkiewicz, K.; Redon, J.; Agabiti Rosei, E.; Mancia, G. Treatment of high blood pressure in elderly and octogenarians: European Society of Hypertension statement on blood pressure targets. Blood Press. 2016, 25, 333-336. [CrossRef]

17. Phillips, R.A.; Xu, J.; Peterson, L.E.; Arnold, R.M.; Diamond, J.A.; Schussheim, A.E. Impact of Cardiovascular Risk on the Relative Benefit and Harm of Intensive Treatment of Hypertension. J. Am. Coll. Cardiol. 2018, 71, 1601-1610. [CrossRef]

18. Lee, J.H.; Choi, J.K.; Jeong, S.N.; Choi, S.H. Charlson comorbidity index as a predictor of periodontal disease in elderly participants. J. Periodontal Implant. Sci. 2018, 48, 92-102. [CrossRef]

19. Lee, S.S.; Ae Kong, K.; Kim, D.; Lim, Y.M.; Yang, P.S.; Yi, J.E.; Kim, M.; Kwon, K.; Bum Pyun, W.; Joung, B.; et al. Clinical implication of an impaired fasting glucose and prehypertension related to new onset atrial fibrillation in a healthy Asian population without underlying disease: A nationwide cohort study in Korea. Eur. Heart J. 2017, 38, 2599-2607. [CrossRef]

20. Kim, D.; Yang, P.S.; Jang, E.; Yu, H.T.; Kim, T.H.; Uhm, J.S.; Kim, J.Y.; Pak, H.N.; Lee, M.H.; Joung, B.; et al. Increasing trends in hospital care burden of atrial fibrillation in Korea, 2006 through 2015. Heart 2018, 104, 2010-2017. [CrossRef]

21. Lee, H.Y.; Yang, P.S.; Kim, T.H.; Uhm, J.S.; Pak, H.N.; Lee, M.H.; Joung, B. Atrial fibrillation and the risk of myocardial infarction: A nation-wide propensity-matched study. Sci. Rep. 2017, 7, 12716. [CrossRef] [PubMed]

22. Verdecchia, P.; Angeli, F.; Reboldi, G. Hypertension and Atrial Fibrillation: Doubts and Certainties From Basic and Clinical Studies. Circ. Res. 2018, 122, 352-368. [CrossRef] [PubMed]

23. Dzeshka, M.S.; Shahid, F.; Shantsila, A.; Lip, G.Y.H. Hypertension and Atrial Fibrillation: An Intimate Association of Epidemiology, Pathophysiology, and Outcomes. Am. J. Hypertens. 2017, 30, 733-755. [CrossRef] [PubMed]

24. Kim, D.; Yang, P.S.; Yu, H.T.; Kim, T.H.; Jang, E.; Sung, J.H.; Pak, H.N.; Lee, M.Y.; Lee, M.H.; Lip, G.Y.H.; et al. Risk of dementia in stroke-free patients diagnosed with atrial fibrillation: Data from a population-based cohort. Eur. Heart. J. 2019, 40, 2313-2323. [CrossRef] 
25. Kim, T.H.; Yang, P.S.; Uhm, J.S.; Kim, J.Y.; Pak, H.N.; Lee, M.H.; Joung, B.; Lip, G.Y.H. CHA2DS2-VASc Score (Congestive Heart Failure, Hypertension, Age $>/=75$ [Doubled], Diabetes Mellitus, Prior Stroke or Transient Ischemic Attack [Doubled], Vascular Disease, Age 65-74, Female) for Stroke in Asian Patients With Atrial Fibrillation: A Korean Nationwide Sample Cohort Study. Stroke 2017, 48, 1524-1530. [CrossRef]

26. Lee,H.; Kim, T.H.; Baek, Y.S.; Uhm, J.S.; Pak, H.N.; Lee, M.H.; Joung, B. The Trends of Atrial Fibrillation-Related Hospital Visit and Cost, Treatment Pattern and Mortality in Korea: 10-Year Nationwide Sample Cohort Data. Korean Circ. J. 2017, 47, 56-64. [CrossRef]

27. Baek, Y.S.; Yang, P.S.; Kim, T.H.; Uhm, J.S.; Park, J.; Pak, H.N.; Lee, M.H.; Joung, B. Associations of Abdominal Obesity and New-Onset Atrial Fibrillation in the General Population. J. Am. Heart Assoc. 2017, 6, e004705. [CrossRef]

28. Song, S.; Yang, P.S.; Kim, T.H.; Uhm, J.S.; Pak, H.N.; Lee, M.H.; Joung, B. Relation of Chronic Obstructive Pulmonary Disease to Cardiovascular Disease in the General Population. Am. J. Cardiol. 2017, 120, 1399-1404. [CrossRef]

(C) 2020 by the authors. Licensee MDPI, Basel, Switzerland. This article is an open access article distributed under the terms and conditions of the Creative Commons Attribution (CC BY) license (http://creativecommons.org/licenses/by/4.0/). 